\title{
PARAMAGNETIC DEFECT CENTERS IN BESOI AND SIMOX BURIED OXIDES
}

\author{
W. L. Warren, M. R. Shaneyfelt, J. R. Schwank, D. M. Fleetwood, and P. S. Winokur \\ Sandia National Laboratories, Albuquerque, NM 87185-5800 \\ France Télécom, Centre National d'Etudes Télécommunications, 38243 Meylan, FRANCE \\ W.P. Maszara and J. B. McKitterick \\ Allied-Signal Aerospace Corporation, Columbia, MD 21045
}

\begin{abstract}
We have combined electron paramagnetic resonance and capacitance-voltage measurements to identify the chemical nature and charge state of defects in BESOI and SIMOX materials. The four types of defect centers observed, charged oxygen vacancies, delocalized hole centers, amorphous-Si centers, and oxygen-related donors, are strikingly similar. In the BESOI materials, the radiation-induced EPR centers are located at or near the bonded interface. Therefore, the bonded interface is a potential hole trap site and may lead to radiation-induced back-channel leakage. In SIMOX materials it is found that all of the defects in the buried oxide are due to excess-Si. Our results using poly-Si/thermal oxide/Si structures strongly suggest that it is the post-implantation, high temperature anneal processing step in SIMOX that leads to their existence. The anneal leads to the outdiffusion of oxygen from the buried oxide creating excess-Si related defects in the oxide and O-related donors in the underlying Si substrates. Last, our study has elucidated a number of interesting aspects regarding the physical nature of a relatively new class of defects in $\mathrm{SiO}_{2}$ : delocalized spin centers. We find that they are hole traps in both SIMOX and BESOI materials.
\end{abstract}

\section{INTRODUCTION}

Two leading techniques for fabricating silicon-oninsulator (SOI) materials are separation by the implantation of oxygen (SIMOX), in which the oxide layer is implanted beneath the surface of a $\mathrm{Si}$ substrate, and bonded and etchback SOI (BESOI), in which two oxidized wafers are bonded together followed by an etchback of one of the Si substrates to a desired thickness. Many studies have shown that the radiation sensitivity of buried oxides is somewhat

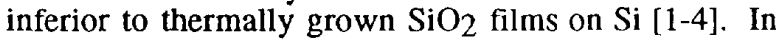
order to obtain a better understanding of the radiation-induced defects in these SOI materials a number of electrical and spectroscopic measurements have been performed [1-15]. For example, Pennise and Boesch [2] have shown that SIMOX materials have a large concentration of deep hole traps and that all of the radiation-induced holes appear to be trapped in place, i.e., there is negligible diffusion before trapping. The work of Stahlbush et al. [1] suggests that many of the defects observed in buried oxide materials are produced by chemically reducing the oxide and producing oxygen vacancies (Si-Si pairs). On the spectroscopic front, a popular tool to investigate the atomic nature of these defects has been electron paramagnetic resonance (EPR). Studies on SIMOX materials by Devine et al. $[7,10]$, Conley, Lenahan and Roitman $[8,11]$, Zvanut et al. [9], and Stesmans and co-workers $[5,13]$ have all shown that charged paramagnetic oxygen vacancies $\left(\mathrm{O}_{3}=\mathrm{Si}^{+}\right.$ - $\mathrm{Si}=\mathrm{O}_{3}$ ) [16], termed $\mathrm{E}_{\gamma}^{\prime}[17]$ centers, can be created by either gamma, $x$-ray, or vacuum ultraviolet (VUV) irradiations. These studies $[8,10]$ have also shown that the $\mathrm{E}_{\boldsymbol{\gamma}}$ center is not the primary source of the induced charge density in the SIMOX materials, and that some of the $E^{\prime}$ centers may be electrically neutral $[8-10]$, i.e., they do not have an accompanying positive charge. A relatively new class of defect centers has been observed in both SIMOX $[13,15]$ and BESOI [12] materials via EPR, and can be categorized as delocalized spin centers. They are delocalized in the sense that the unpaired electron is not associated with any one particular atom. The delocalized center in SIMOX was first identified by Stesmans and coworkers [5,13]; they attributed the defect to a delocalized type of $\mathrm{E}^{\prime}$ center, termed the $\mathrm{E} \delta$ ' center. In their model the delocalized spin of the $\mathrm{E} \delta^{\prime}$ center is based on the existence of clusters of $5 \mathrm{Si}$ atoms in the buried oxide [13]. They were further able to show that the $\mathrm{E}_{\delta}^{\prime}$ center is not associated with $\mathrm{Cl}$ or $\mathrm{F}$, as first tentatively suggested by others working in bulk fused silica [18]. Devine et al. [19] were able to show that $\mathrm{E}_{\delta}{ }^{\prime}$ centers can also be found in poly-Si/thermal $\mathrm{SiO}_{2} / \mathrm{Si}$ structures that received a high temperature post-oxidation anneal. In the BESOI materials, another type of delocalized spin center was found, and was termed the EH center [12]. The EH center was categorized as a hydrogen-related delocalized spin center.

In this study we build upon the previous work by relating the various paramagnetic defects created by VUV irradiations or selective hole injection to the nature of the positive charge generated in SIMOX, BESOI and poly-Si/thermal $\mathrm{SiO}_{2} / \mathrm{Si}$ structures. The defects examined include the $\mathrm{E}_{\gamma^{\prime}}, \mathrm{E}^{\prime}, \mathrm{EH}$, amorphous $\mathrm{Si} D$ centers $\left(\cdot \mathrm{Si}_{\mathrm{S}} \mathrm{Si}_{3}\right)$ in the oxide layer, and $\mathrm{O}$ - 
related donors in the $\mathrm{Si}$ substrates. We demonstrate that the delocalized spin centers in SIMOX $\left(E_{\delta}^{\prime}\right)$ and BESOI (EH) are positively charged when paramagnetic. We have also been able to show that the classic $\mathrm{E}_{\gamma}\left(\mathrm{O}_{3} \equiv \mathrm{Si} \cdot+\mathrm{Si} \equiv \mathrm{O}_{3}\right)$ and $\mathrm{D}(\mathrm{Si} \equiv \mathrm{Si} \bullet)$ centers in these buried oxides are also most likely positively charged when paramagnetic. The paramagnetic defects in the oxide of the BESOI materials are all located at or near the bonded interface, making this interface susceptible to radiation damage. On a more general note, our results demonstrate that the presence of excess-Si in the buried oxide materials may dominate its charge trapping characteristics. These results also strongly suggest that the "inert" post-oxidation anneal of the buried oxides leads to the degradation of SOI devices by creating $\mathrm{O}$-deficiencies in the $\mathrm{SiO}_{2}$ layer and excess-O in the underlying (or overlying) $\mathrm{Si}$ substrates.

In this paper, we will first discuss the defects induced by hole injection or $x$-ray irradiation in BESOI materials. Next, defects in SIMOX buried oxides will be presented, followed by our investigation of poly-Si/thermal oxide/Si structures that have either received a high temperature post-oxidation anneal $\left(1320^{\circ} \mathrm{C}\right.$ for $6 \mathrm{hrs}$ in $\left.99 \% \mathrm{Ar} / 1 \% \mathrm{O}_{2}\right)$ or have not. Finally we will compare the nature of the paramagnetic centers in BESOI materials to those observed in SIMOX and in the poly-Si/thermal oxide/Si structures. Implications for the radiation hardness of SOI technology are discussed.

\section{EXPERIMENTAL DETAILS}

Two sets of BESOI materials were prepared. The first set was fabricated by growing a $0.055 \mu \mathrm{m}$ oxide at $850^{\circ} \mathrm{C}$ in $\mathrm{O}_{2}$ on the seed wafer (top oxide) and a $1.07 \mu \mathrm{m}$ oxide at $900^{\circ} \mathrm{C}$ in $\mathrm{O}_{2}$ on the handle wafer (bottom oxide). The two wafers were bonded together at $900^{\circ} \mathrm{C}$ for $10 \mathrm{~min}$. in $\mathrm{N}_{2}$. After bonding the wafers together, the seed wafer was etched to leave a thin 0.4 $\mu \mathrm{m}) \mathrm{Si}$ layer [20]. Both starting $\mathrm{Si}$ substrates were Czochralski p-type (100) wafers. The second set was fabricated by growing a $0.045 \mu \mathrm{m}$ oxide at $850^{\circ} \mathrm{C}$ in wet oxygen on the seed wafer and a $0.865 \mu \mathrm{m}$ oxide at $900^{\circ} \mathrm{C}$ in wet oxygen for $12 \mathrm{hrs}$ with TCA on the handle wafer. The two wafers were bonded together at $900^{\circ} \mathrm{C}$ for $10 \mathrm{~min}$. in $\mathrm{N}_{2}$. The starting substrates were (100) p-type float zone Si wafers. The float zone wafers were investigated to ensure that the source to create the O-related donors in the substrate did not come from the O-rich Czochralski wafers. The seed wafer in this set did not have a built-in etch stop, hence for these samples the top Si layer was etched off following the bonding procedure. To explore the effects of the bonding process, EPR measurements for the bonded wafers were compared to EPR measurements for the non-bonded, oxidized float zone handle wafers.
Single implant SIMOX wafers were made by implanting $10^{18} \mathrm{O}^{+} / \mathrm{cm}^{2}$ at an energy of $190 \mathrm{keV}$ into p-type (100) wafers held at $600^{\circ} \mathrm{C}$. After the implantation step, the wafers were annealed at $1320^{\circ} \mathrm{C}$ for $6 \mathrm{hrs}$ in a 99\% Ar and $1 \% \mathrm{O}_{2}$ ambient [21]. The EPR measurements provide information regarding the atomic nature of defects; however, it is imperative to know the spatial location and charge state of the defects to understand their potential roles in radiation damage. To do this, CV measurements, etchback experiments, and selective hole and electron injection experiments were coupled with the EPR results. Prior to performing etchback of the $\mathrm{SiO}_{2}$, the top Si layer of the BESOI and SIMOX wafers was etched off in a $10 \% \mathrm{KOH}$ solution at $50^{\circ} \mathrm{C}$.

Thermal oxides for the poly- $\mathrm{Si} / \mathrm{SiO}_{2} / \mathrm{Si}$ structures were grown in dry $\mathrm{O}_{2}$ at $1050^{\circ} \mathrm{C}$ to a thickness of $0.41 \mu \mathrm{m}$. $200 \mathrm{~nm}$ poly-Si films were deposited on the oxide from $\mathrm{SiH}_{4}$ gas using low pressure chemical vapor deposition at $620^{\circ} \mathrm{C}$. Further details can be found in ref. [19]. A batch of these samples were annealed at $1320^{\circ} \mathrm{C}$ for $6 \mathrm{hrs}$ in a $99 \% \mathrm{Ar} / 1 \% \mathrm{O}_{2}$ ambient. (This is identical to the SIMOX anneal.) Following the anneal the poly-Si layer was removed by etching in $\mathrm{XeF}_{2}$ gas. Unannealed reference samples were produced by undergoing exactly the same production steps except for the $1320^{\circ} \mathrm{C}$ anneal.

The EPR measurements were performed on an Xband Bruker spectrometer between $4.2 \mathrm{~K}$ and $300 \mathrm{~K}$. A frequency counter in conjunction with a MicroNow NMR Gaussmeter was used to accurately determine the g-values. Spin densities were determined by comparing the double integrated intensity of a weak pitch or ruby standard to the samples under investigation. The absolute spin densities are accurate to within about a factor of two; the relative spin densities are accurate to within approximately $15 \%$ primarily since some of the EPR lines overlap. Low temperature EPR measurements were made to enhance some of the defects that are broad, have a shorter spin lattice relaxation time, or can only be occupied, and hence, paramagnetic at low temperature (O-related donors). To apply an electric field to the structures in situ in the EPR cavity, a corona discharge apparatus was employed. The positive or negative corona ion bias was determined using a Monroe Electronics Kelvin probe electrostatic voltmeter.

A vacuum ultraviolet (VUV) source was used in conjunction with positive corona ions to selectively inject holes into the oxides and apply a bias. Electrons were internally photo-injected from the $\mathrm{Si}$ substrate into the $\mathrm{SiO}_{2}$ using a UV lamp (hv $\left.<5.5 \mathrm{eV}\right)$ and positive corona ions. Standard CV measurements were made using a $\mathrm{Hg}$ probe. To determine the spatial location of the EPR centers and charge centers sensed by $\mathrm{CV}$, etchback experiments were performed on the $\mathrm{SiO}_{2}$. The etchant in this case was a buffered HF solution. 


\section{RESULTS AND DISCUSSION}

\section{A. BESOI}

Figure 1 illustrates EPR spectra for the BESOI materials. Trace 1(a) is for the virgin samples. Trace 1(b) was taken following an unbiased, unfiltered VUV illumination (hy $<10.2 \mathrm{eV}$ ) for $3 \mathrm{hrs}$. Trace $1(\mathrm{c})$ was taken after illuminating for $12 \mathrm{hrs}$. It is quite clear that the VUV illumination generates more $\mathrm{E}^{\prime}$ centers and a broader signal at $g=2.0042$. The broader signal may be attributed to the amorphous silicon center $(\cdot \mathrm{Si} \equiv \mathrm{Si} 3)$ by noting that the g-value is characteristic of dangling bonds in amorphous silicon prepared by various means; this signal is typically classified as the D-center with reported $\mathrm{g}$ values from 2.0041 to 2.0061 [22]. Even though the $g=2.0042$ signal has been attributed to a D-center, the g-value is not the typically quoted $\mathrm{g}$ $=2.0055$. It may be that the unpaired spin on the $S i$ atom is bonded to $2 \mathrm{Si}$ atoms and $1 \mathrm{O}$ atom to shift the $\mathrm{g}$-value down from 2.0055. Further work is needed to accurately identify this center. Figure 1 also shows a very narrow $(1.1 \mathrm{G})$ isotropic signal at $\mathrm{g}=2.0025$ along with its associated hyperfine lines split by $23.1 \mathrm{G}$. The spin densities for the $E^{\prime}$ center and the narrow signal at $\mathrm{g}=2.0025$ are $4 \times 10^{12} / \mathrm{cm}^{2}$ and $6 \times 10^{11} / \mathrm{cm}^{2}$, respectively.

As discussed elsewhere [12], this narrow line centered at $\mathrm{g} \approx 2.0025$ ( $1.1 \mathrm{G}$ peak-to-peak line width) has been observed in $\mathrm{SiO}_{2}$ by a number of other investigators $[5,13,18,23,24]$. Griscom and Friebele [18] and Stesmans [23] have provided convincing evidence that this defect center is a delocalized spin by observing its corresponding ${ }^{29} \mathrm{Si}$ hyperfine interactions. It was suggested earlier [12] that the hyperfine interaction arises from the presence of hydrogen not ${ }^{29} \mathrm{Si}$.

To the best of our knowledge, a defect with this hyperfine coupling constant has never been observed in $\mathrm{SiO}_{2}$. We term this center the EH center. Hydrogen is very mobile in $\mathrm{SiO}_{2}$ and can easily explain the hyperfine to central line ratio increase with VUV irradiation time as indicated in Fig. 1. Likewise, the observed ratio being as high as 0.48 cannot be explained in terms of equivalent ${ }^{29} \mathrm{Si}$ neighbors. It is interesting to note that different $\mathrm{H}$-related $\mathrm{E}^{\prime}$ centers, termed the $74 \mathrm{G}$ and $10.4 \mathrm{G}$ doublets, have also been observed in SIMOX materials [25].

It was suggested earlier [12] that the hyperfine interaction arises from the presence of hydrogen not ${ }^{29} \mathrm{Si}$. To the best of our knowledge, a defect with this hyperfine coupling constant has never been observed in $\mathrm{SiO}_{2}$. We term this center the $\mathrm{EH}$ center. Hydrogen is very mobile in $\mathrm{SiO}_{2}$ and can easily explain the hyperfine to central line ratio increase with VUV irradiation time as indicated in Fig. 1. Likewise, the observed ratio being as high as 0.48 cannot be explained in terms of equivalent ${ }^{29} \mathrm{Si}$ neighbors. It is interesting to note that different $\mathrm{H}$-related $\mathrm{E}^{\prime}$ centers termed the $74 \mathrm{G}$ and $10.4 \mathrm{G}$ doublets, have also been observed in SIMOX materials [25].

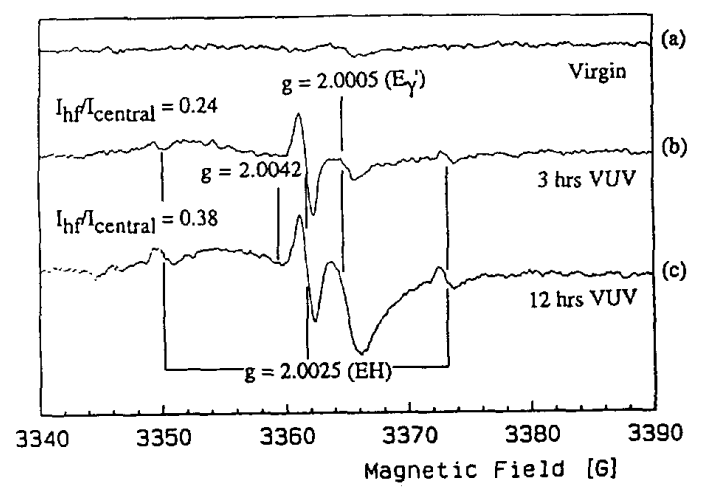

Fig. 1 Low temperature (20K) EPR traces for BESOI oxides: (a) unirradiated, and VUV irradiated for (b) 3 hrs, and (c) $12 \mathrm{hrs}$. After ref. [12].

It was suggested earlier [12] that the hyperfine interaction arises from the presence of hydrogen not ${ }^{29} \mathrm{Si}$. To the best of our knowledge, a defect with this hyperfine coupling constant has never been observed in $\mathrm{SiO}_{2}$. We term this center the EH center. Hydrogen is very mobile in $\mathrm{SiO}_{2}$ and can easily explain the hyperfine to central line ratio increase with VUV irradiation time as indicated in Fig. 1. Likewise, the observed ratio being as high as 0.48 cannot be explained in terms of equivalent ${ }^{29} \mathrm{Si}$ neighbors. It is interesting to note that different $\mathrm{H}$-related $\mathrm{E}^{\prime}$ centers, termed the $74 \mathrm{G}$ and $10.4 \mathrm{G}$ doublets, have also been observed in SIMOX materials [25].

Figure 2 shows the isochronal anneal characteristics of the $E_{\gamma}$ and $E H$ centers. This figure is reproduced from ref. [12]. The anneals were performed in air for $15 \mathrm{~min}$. at each temperature. The EH center is significantly less stable than the E $y$ center. This is in accord with the localized nature of the $E_{\gamma}$ center; whereas, the EH center is a delocalized spin center (spread over many atoms, some of which are hydrogen) and is not strongly bound to any one atom.

Figure 3 illustrates the EPR-response to the selective injection of electrons or holes into the buried oxide films. Trace $3(\mathrm{~b})$ shows that hole injection (10 14 holes $/ \mathrm{cm}^{2}$ ) creates $E^{\prime}$ centers and EH centers; this strongly suggests that these two defect centers are positively charged when paramagnetic (EPR-active). It is thus reasonable to assume that the $E_{\gamma}^{\prime}$ centers are holes trapped at oxygen vacancies, i.e., $\mathrm{O}_{3} \equiv \mathrm{Si} \cdot{ }^{+} \mathrm{Si} \equiv \mathrm{O}_{3}$ [16]. In contrast, as illustrated in Trace 3(c), these paramagnetic centers could not be generated by electron injection $\left(10^{15}\right.$ electrons $\left./ \mathrm{cm}^{2}\right)$; the injection was performed by photoinjecting electrons from the bottom $\mathrm{Si}$ substrate into the $\mathrm{SiO}_{2}$ using a UV lamp (hV $<5.5 \mathrm{eV}$ ) and a positive bias. The broad $\mathrm{D}$ center was 
not observed in either of these carrier injection remaining oxide thickness, indicating a uniform experiments; this may be due to the rather small distribution of the positive space charge. (Although number of carriers injected. Last, to explore the effects not shown in Fig. 4 , the spatial location of the positive of the bonding process, we also injected holes into the charge in the oxidized handle wafer is essentially oxidized handle wafers as illustrated in Trace $3(\mathrm{~d})$; identical to that observed in BESOI. Likewise, the Dneither $\mathrm{E}_{\gamma}$ nor $\mathrm{EH}$ centers were created by hole center observed in Fig. 1 is located near the top $\mathrm{Si}_{\mathrm{SiO}} 2$ injection into the oxidized handle wafers.

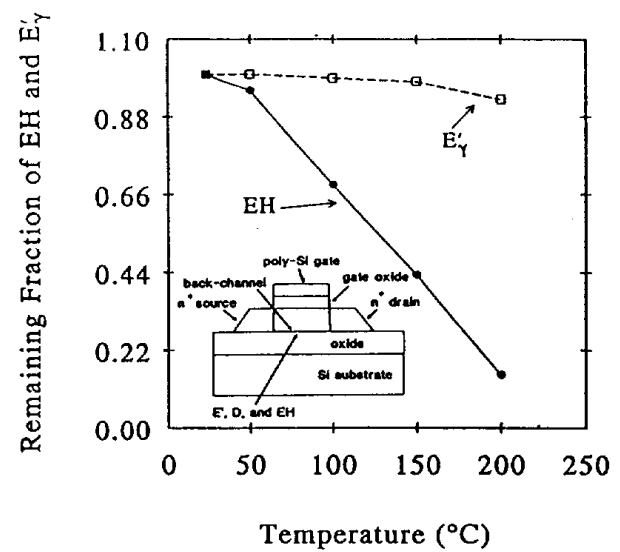

Fig. 2 Isochronal anneal characteristics for the $\mathrm{EH}$ and $\mathrm{E}_{\boldsymbol{\gamma}}$ centers. The inset schematically illustrates a SOI transistor and the spatial locations of the EPR defects in BESOI materials. After ref. [12].

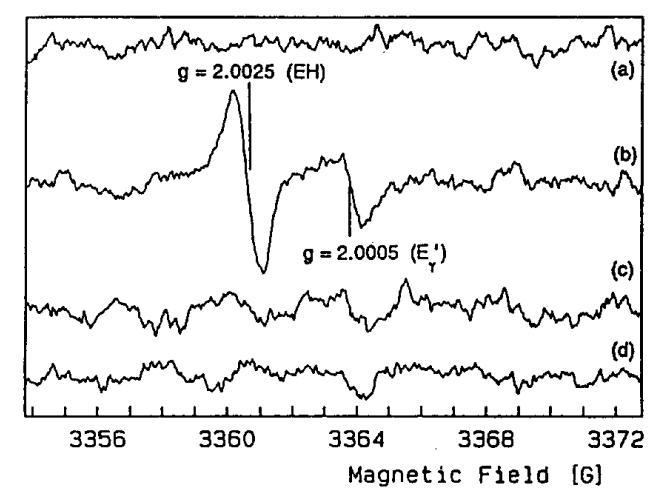

A number of considerations become apparent from these results. First, as illustrated in Fig. 4, most of the net positive charge observed by $\mathrm{CV}$ is not associated with any of the EPR centers. Second, of technological interest, the bond between the original oxides is 0.055 $\mu \mathrm{m}$ from the top $\mathrm{Si} / \mathrm{SiO}_{2}$ interface as are most of the EPR centers, making this interface more susceptible to instabilities that may arise from their presence. Of technological interest, the notion that the bonded interface is where most of the EPR-active centers lie can be partially substantiated by noting that $\mathrm{E}_{\gamma^{\prime}}$ and $\mathrm{EH}$ centers were not created in the oxidized handle wafers (Fig. 3(d)).

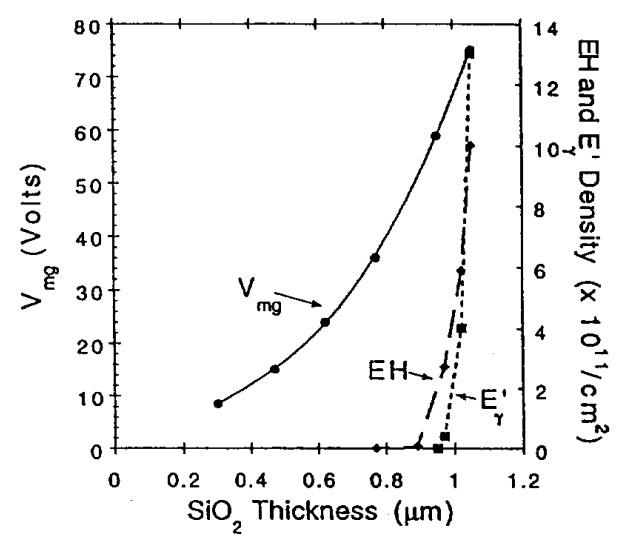

Fig. 4. Etchback experiments of the midgap voltage, $v_{m g}$, and $E H$ and $\mathrm{E}_{\gamma}$ centers in BESOI oxides that were injected with holes.

A number of considerations become apparent from these results. First, as illustrated in Fig. 4, most of the net positive charge observed by $\mathrm{CV}$ is not associated with any of the EPR centers. Second, of technological interest, the bond between the original

Fig. 3 Room temperature EPR traces for (a) virgin, (b) hole injected, and (c) electron injected BESOI oxides. Trace (d) is for the oxidized handle wafer subjected to hole injection.

Having identified paramagnetic signals created in BESOI materials, it is of interest to determine their location and charge state(s). To determine the location of the trapped charge and paramagnetic defect centers, etchback experiments were performed. The results are illustrated in Fig. 4. $(0.0 \mu \mathrm{m}$ corresponds to the bottom $\mathrm{Si} / \mathrm{SiO}_{2}$ interface.) It is quite interesting that the EPR signals are located at or near the top interface region. In contrast, $C V$ measurements show a net positive space charge density of $3 \times 10^{12} / \mathrm{cm}^{2}$ that is located throughout the buried oxide; the midgap voltage shift varies approximately as the square of the is $0.055 \mu \mathrm{m}$ from the top $\mathrm{Si} / \mathrm{SiO}_{2}$ interface as are most of the EPR centers, making this interface more susceptible to instabilities that may arise from their presence. Of technological interest, the notion that the bonded interface is where most of the EPRactive centers lie can be partially substantiated by noting that $\mathrm{E}_{\gamma}$ and $\mathrm{EH}$ centers were not created in the oxidized handle wafers (Fig. 3(d)).

Furthermore, Boesch and Taylor [4] have shown that there is enhanced hole trapping at this top $\mathrm{Si} / \mathrm{SiO}_{2}$ interface. Similarly, we have shown that the E $\gamma$ ' and $\mathrm{EH}$ centers are also located at or near this top interface, and are most likely positively charged. This top interface corresponds to the back-channel in actual device structures, schematically illustrated in the 
inset of Fig. 2. Enhanced hole trapping may lead to significant back-channel leakage in a radiation environment. These observations are not limited to the VUV illuminations or hole injection experiments. Identical observations regarding the location of the EPR centers and space charge were made in the BESOI samples subjected to $x$-ray irradiation. In this case, it is known that the $\mathrm{x}$-rays create electron/hole pairs uniformly throughout the dielectric. We find that the $E_{H}$ and $E_{\gamma}^{\prime}$ centers are created by selective hole injection, and are annihilated by subsequent electron injection. This strongly suggests that they are positively charged when paramagnetic, i.e., the EH defect is a delocalized hole center, and the $E_{\gamma}^{\prime}$ centers are holes trapped at oxygen vacancies.

We have also been able to observe another defect in the BESOI materials which we attribute to a shallow $\mathrm{O}$-related donor in the silicon. Figure 5 illustrates the electric field modulated EPR spectra for unirradiated BESOI materials. The samples used in Fig. 5 were made from float zone $\mathrm{Si}$ substrates. The spectra (Trace 5 (b)) show that an EPR-signal is activated by applying a positive bias $(+20 \mathrm{~V})$ to the oxide surface, i.e., a positive "gate" bias. The small donor signal observed in Trace 5(a) probably results from a small amount of fixed positive charge in the oxide layer and/or a small positive built-in field due to the Si work function. As shown in Trace 5(c), no signal was observed with negative corona ions $(-25 \mathrm{~V})$. When the magnetic field is aligned along the [100] direction the $\mathrm{g}$ value is 1.99994. In order to aid in defect identification, and to determine the symmetry of the defect, the sample was rotated in the EPR cavity. The g-value anisotropy map for this EPR signal on (100) $\mathrm{Si}$ substrates at different angles of rotation of the magnetic field in the $(011)$ plane indicated that the center exhibited axial symmetry with $\mathrm{g}_{\|}=1.99994$ and $\mathrm{g}_{\perp}=1.99958 \pm 0.00007$.

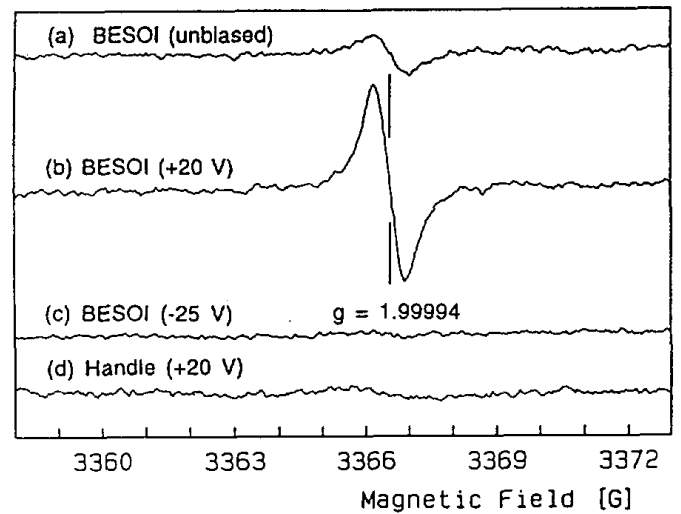

Fig. 5 Low temperature (15 K) EPR traces for (a) virgin, (b) positively corona biased, and (c) negatively biased BESOI oxides. (d) Positively biased oxidized handle wafer processed identically to the BESOI materials.
From these collective observations it can now be argued that the EPR signal shown in Fig. 5 is due to donors by considering band bending effects at the $\mathrm{Si} / \mathrm{SiO}_{2}$ interface. Under the flat band or negative gate bias conditions the donor level $\left(\mathrm{E}_{\mathrm{d}}\right)$ is above the Fermi level $\left(E_{f}\right)$. Under these conditions the donor level will not be occupied, and hence EPR-inactive. However, under positive bias conditions, $E_{f}$ will lie above $E_{d}$ making the donor level occupied, and EPRactive, in agreement with our observations. It should be noted that the electric field response of these donors indicates that most of the EPR-active centers must be spatially located within the depletion width of the "capacitor" structure $(1 \mu \mathrm{m})$. The donors observed in this study were determined to be located at the bottom $\mathrm{Si}$ substrate, near the bottom $\mathrm{Si} / \mathrm{SiO}_{2}$ interface. Recall that the top $\mathrm{Si}$ layer was etched during the BESOI processing since the seed wafer did not have a built-in etch stop, hence the top interface could not be investigated.

Having in part established the donor nature of the defect, we now suggest that the donor is most likely $\mathrm{O}$ related. Spectroscopic evidence for this includes (1) that its $\mathrm{g}$-value is close to that for $\mathrm{O}$ donors in $\mathrm{Si}$ $[5,14,26-29]$ and (2) that its g-tensor anisotropy is very close to that observed for O-related donors in oxygen-rich $\mathrm{Si}$ [26-29]. The g-tensors are not consistent with vacancy-related defects in $\mathrm{c}-\mathrm{Si}$ substrates [29]. Since substitutional donors such as $\mathbf{P}$, $\mathrm{As}$, and $\mathrm{Sb}$ are isotropic in $\mathrm{Si}$ [30], these donor species can be ruled out. Also, conduction electrons can be ruled out since their $g$ value is isotropic and equal to 1.99875 [30]. The lineshape and intensity dependence on temperature also strongly indicate that this defect is a donor, i.e., the intensity follows a Curie-Weiss law (not shown).

O-related donors, termed UL1, have also been recently observed in SIMOX materials by Stesmans and co-workers $[5,14,28]$. The O-related donor observed in our study is very similar to the one observed in SIMOX materials and may arise from a similar phenomenon. More will be said about this defect in SIMOX later.

To determine if this $\mathrm{O}$-related donor is related to the oxidation or bonding process in BESOI materials, we have also examined float zone handle wafers with $0.865 \mu \mathrm{m}$ thermally grown oxides. As shown in Trace 5(d), we find no donor signal in the handle wafer, thus strongly suggesting that the defect is associated with the bonding process. Following the recent work of Devine et al. [19], we suggest that the donor results from the $900^{\circ} \mathrm{C} \mathrm{N} 2$ post-oxidation anneal during the bond formation. In their work [19] it was shown using EPR and diffusion theory that O-related donors result from the diffusion of $\mathrm{O}$ from the $\mathrm{SiO}_{2}$ network into the $\mathrm{Si}$ substrate when poly- $\mathrm{Si} / \mathrm{SiO}_{2} / \mathrm{Si}$ structures are given a high-temperature, post-oxidation, nonoxidizing anneal. Recall that float zone Si substrates were used to ensure that the source of $\mathrm{O}$ did not come from the $\mathrm{Si}$; thus, the only reasonable possibility is 
that it came from the buried $\mathrm{SiO}_{2}$ layer. Because the donors are related to the anneal during the bonding process, we expect that the donors would also be present at the technologically important top $\mathrm{Si} / \mathrm{SiO}_{2}$ interface. However, further work is needed to support this expectation. In any event, these donors will make it difficult to predict the doping density in the underlying Si substrates.

\section{B. SIMOX}

Turning now to defects in SIMOX, we find, as illustrated in Fig. 6, that an unbiased, unfiltered VUV illumination generates a large $E^{\prime}$ signal without a large accompanying shift in the $\mathrm{CV}$ curve $(+3 \mathrm{~V}$ ) (not shown). This was also observed by Conley, Lenahan and Roitman $[8,11]$ and Herve, Leray and Devine [10]; we simply reproduce these results to make a better comparison with the BESOI materials and the poly-Si/thermal $\mathrm{SiO}_{2} / \mathrm{Si}$ structures. The spatial location of the $E^{\prime}$ centers is shown in Fig. 7. The $E^{\prime}$ centers are distributed throughout the buried oxide layer.

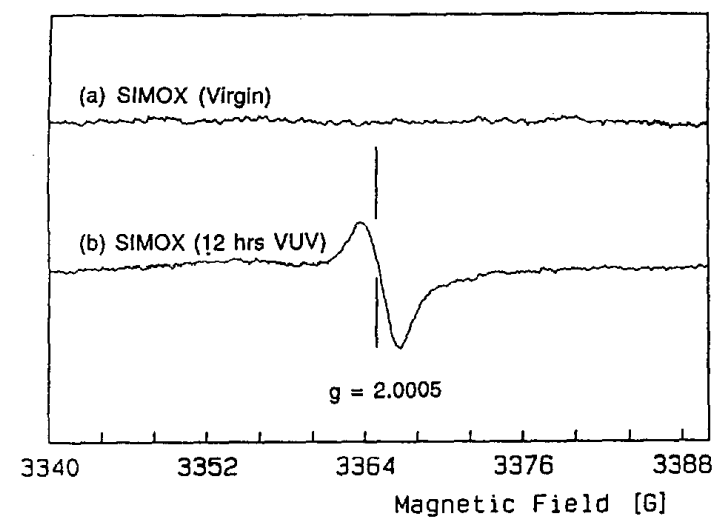

Fig. 6. Low temperature $(20 \mathrm{~K})$ EPR traces of $\mathrm{E}_{\gamma}$ centers in (a) virgin, and (b) VUV irradiated SIMOX wafers. In this trace the $\mathrm{E}_{\gamma}$ ' center is overmodulated, hence, the axially symmetric lineshape is not exhibited.

Contrary to the unbiased, unfiltered VUV illumination case, we found [15] that hole injection creates three types of EPR defects: $E_{\gamma^{\prime}}, \mathrm{E}_{\delta^{\prime}}$ and Dcenters, and a rather large negative shift in the CV curve $(-85 \mathrm{~V})$. To make a direct comparison with the BESOI wafers and the poly-Si/thermal oxide/Si structures, the creation of $\mathrm{E}_{\gamma^{\prime}}$ and $\mathrm{E}_{\delta}^{\prime}$ centers by hole injection and their subsequent annihilation by electron injection are reproduced from ref. [15] in Fig. 8. If the samples are cooled to $20 \mathrm{~K}$ following hole injection, a broad signal around $\mathrm{g}=2.0061$ is observed as shown in Fig. 9. The $\mathrm{E}_{\gamma^{\prime}}$ and $\mathrm{E}_{\delta}{ }^{\prime}$ centers are overmodulated and saturated in Fig. 9, and are hence, substantially smeared out. (Fig. 9 is adapted from ref. [15].) The $\mathrm{E} \gamma^{\prime}, \mathrm{E} \delta^{\prime}$ and $\mathrm{D}$ centers are only created by hole injection; they are not created by electron injection. Therefore, it is reasonable to assume that they are positively charged when paramagnetic. Similar observations regarding the positive charge state of the Ey center in SIMOX have been demonstrated in the studies of Conley, Lenahan, and Roitman $[8,11]$ and Herve, Leray, and Devine [10]. As shown elsewhere [15], the spatial locations of the positive charge, $\mathrm{E}_{\gamma}^{\prime}$ and $\mathrm{E}_{\delta}^{\prime}$ centers exhibit similar spatial locations throughout the buried oxide consistent with their positive charge assignment. In contrast, the D-centers are primarily located near the top $\mathrm{Si} / \mathrm{SiO}_{2}$ interface. It was also shown [15] that there is a discrepancy between the positive charge density and the density of $E^{\prime} \gamma, E^{\prime} \delta$ and D-centers. The discrepancy was also observed by other investigators [8-11] examining the relationship between $\mathrm{E}_{\gamma^{\prime}}$ and space charge.

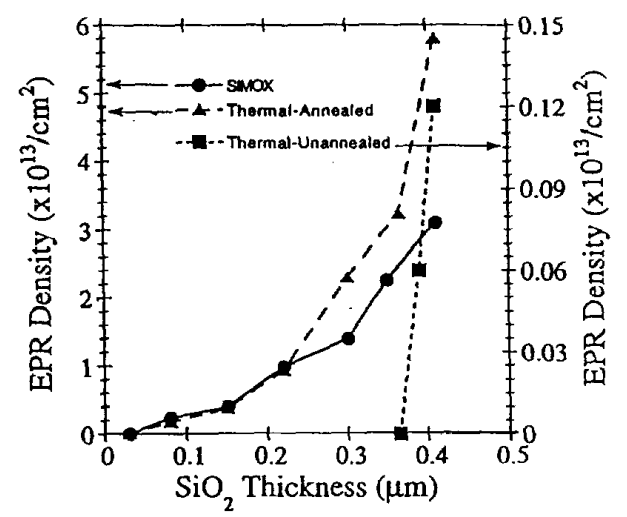

Fig. 7 Etchback experiments illustrating the locations of the $E^{\prime}$ centers created in SIMOX, and annealed, and unannealed poly$\mathrm{Si} /$ thermal oxide/Si strucures subjected to an unbiased, unfiltered VUV irradiation.

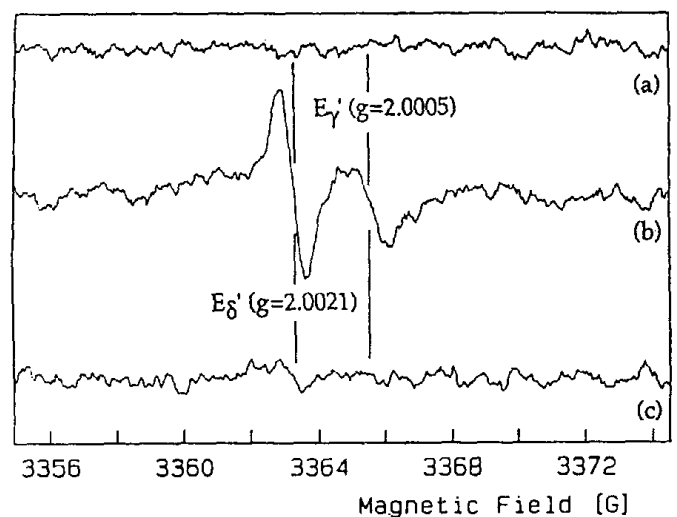

Fig. 8 Room temperature SIMOX EPR: (a) virgin, (b) bole, and (c) subsequent electron injection. After ref. [15]. 


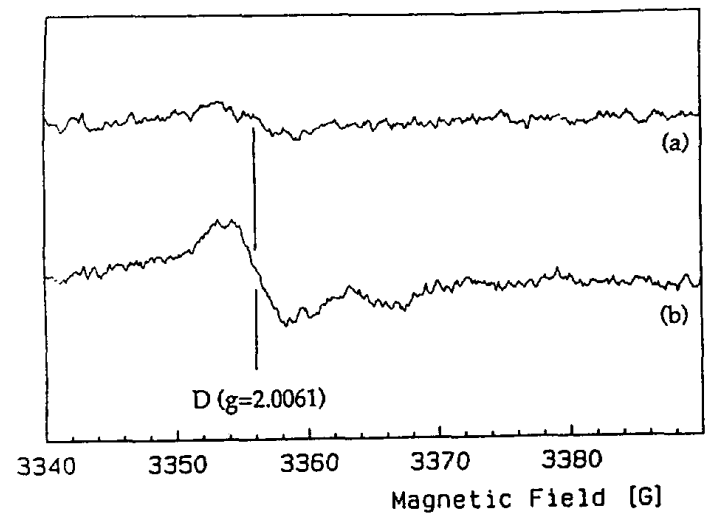

Fig. 9 Low temperature $(20 \mathrm{~K})$ SIMOX EPR of D-centers in (a) virgin and (b) bole injected wafers. After ref. [15].

Figure 10 shows the isochronal anneal characteristics of the $\mathrm{E}_{\gamma}$ and $\mathrm{E}_{\delta}^{\prime}$ centers. The anneals were performed in air for $15 \mathrm{~min}$. at each temperature. Again, like the $\mathrm{EH}$ center in the BESOI materials, the $\mathrm{E} \delta^{\prime}$ center is significantly less stable than the $\mathrm{E}_{\boldsymbol{\gamma}}$ center.

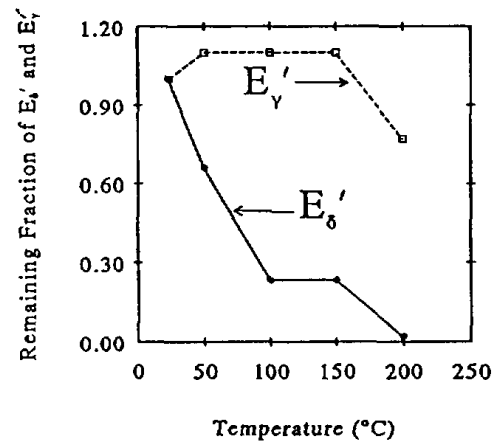

Fig. 10 Isochronal anneal characteristics of the $E_{\gamma}$ and $E_{\delta}$ centers in SIMOX. The anneals were performed in air for $15 \mathrm{~min}$ at each temperature.

It is somewhat peculiar that the $\mathrm{E}_{\delta}$ centers are created by hole injection but are not observed after VUV unbiased, unfiltered irradiation, or with $\mathrm{x}$-ray irradiations under either a positive or negative gate bias [10]. We believe that this may arise because the paramagnetic $E_{\delta}^{\prime}$ center has a rather large capture cross-section for electrons as compared to the paramagnetic $\mathbf{E}_{\gamma^{\prime}}$ center. For the case in which electrons and holes are simultaneously generated by the irradiation, once the center has captured a hole becoming paramagnetic, it can quickly capture an electron again becoming neutral and diamagnetic. When holes are selectively injected, the $\mathrm{E} \delta^{\prime}$ can be observed since accompanying electrons are not generated. To partially support this notion, we find that following hole injection the $E \delta^{\prime}$ centers are annihilated by electrons at a much faster rate than the $\mathrm{E}_{\gamma}$ centers (not shown).

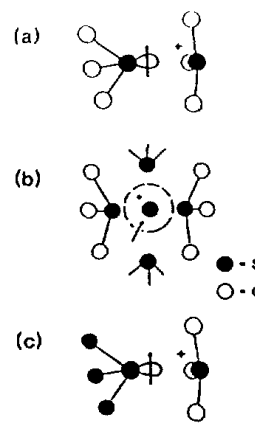

Figure 11 schematically illustrates models for the (a) $\mathrm{E}_{\gamma}^{\prime}[16,17]$, (b) $\mathrm{E}_{\delta}[13,18]$, and (c) D centers. As discussed earlier, like the $\mathrm{EH}$ center, the $\mathrm{E}_{\delta^{\prime}}$ center is also a delocalized spin center.

One notes from Fig. 11 that all of the paramagnetic defects in SIMOX are excess-Si related. The existence of these Si-related defects probably results from a competition between the oxidation (from the implanted oxygen) and $\mathrm{O}$ out-diffusion (from the high temperature anneal) of the SIMOX process as will be discussed in greater length later.

\section{Poly-Si/SiO2/Si STRUCTURES}

In order to help understand the effects of the high temperature processing and the role of the top $\mathrm{Si}$ layer, poly-Si/thermal $\mathrm{SiO}_{2} / \mathrm{Si}$ structures were investigated. One set was annealed at $1320^{\circ} \mathrm{C}$ for $6 \mathrm{hrs}$ in a $99 \% \mathrm{Ar} / 1 \% \mathrm{O}_{2}$ ambient (identical to the SIMOX anneal). The other set did not receive the $1320^{\circ} \mathrm{C}$ anneal. The effects of an unbiased, unfiltered VUV illumination for the annealed and unannealed samples are illustrated in Fig. 12. The irradiated annealed samples exhibit a significantly larger $\mathrm{E}^{\prime}$ defect density than the unannealed samples. It should be stressed that the CV curve of the annealed sample subjected to this irradiation had very little net space charge $(+3.5$ $\mathrm{V})$ like the SIMOX $(+3 \mathrm{~V})$ samples; whereas, the CV curve of the unannealed sample shifted $-32 \mathrm{~V}$. The corresponding spatial locations of the $E^{\prime}$ centers in the annealed and unannealed thermal oxide structures are shown in Fig. 7. As shown, the spatial location and density of $\mathrm{E}^{\prime}$ centers for the annealed $\mathrm{Si} /$ thermal oxide/Si structures are close to what is observed in SIMOX. 


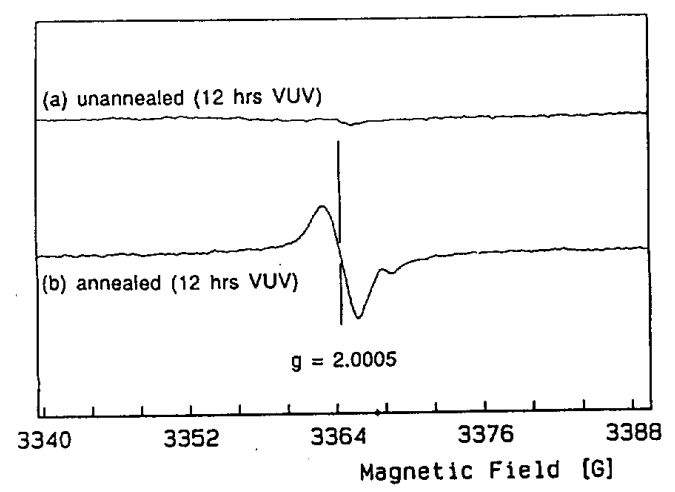

Fig. 12. Low temperature $(20 \mathrm{~K})$ EPR spectra of $\mathrm{E}_{\gamma}$ centers in VUV irradiated (a) unannealed and (b) annealed poly-Si/thermal oxide/Si structures. The $\mathrm{E}_{\boldsymbol{\gamma}}$ centers are overmodulated in these traces.

Although not the focus of this paper, it should be noted that the positive space charge in the unanealed structures was located near the bottom $\mathrm{Si} / \mathrm{SiO}_{2}$ interface, $\left(1.7 \times 10^{12} / \mathrm{cm}^{2}\right)$ opposite to the interface in which the $\mathrm{E}^{\prime}$ centers are located as illustrated in Fig. 7.

Figure 13 shows the effects of selective hole injection into the annealed and unannealed oxide structures. Hole injection activates both $\mathrm{E}_{\gamma^{\prime}}$ and $\mathrm{E}_{\boldsymbol{\delta}}{ }^{\prime}$ centers as was also found in SIMOX materials. (The defect density in the annealed samples is again larger than that found in the unannealed samples. The hole injection results do not allow us to determine with great accuracy the saturation of defects because of the buildup of an internal field induced by the trapping of the charge injected.) Just as observed in SIMOX materials, the $\mathrm{E}_{\delta}$ centers could only be activated by hole injection; the unbiased, unfiltered VUV illumination only created $\mathrm{E}_{\gamma}$ centers.

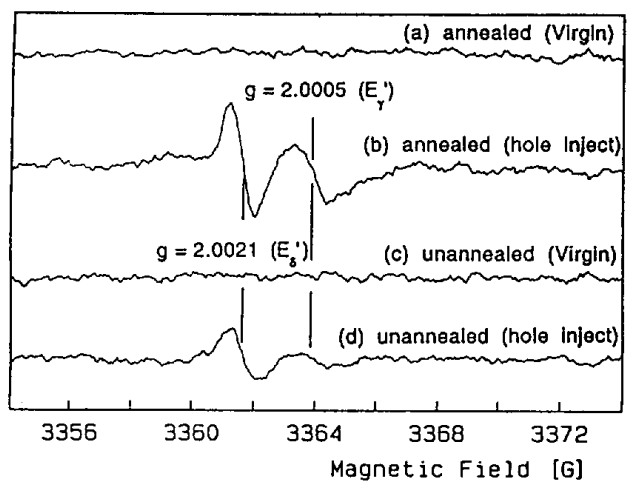

Fig. 13. Room temperature EPR traces of $E_{y}$ and $E_{\delta} \delta^{\prime}$ centers in (a) virgin, and (b) hole injected annealed poly-Si/thermal oxide/Si structures. Traces (c) and (d) are for virgin and bole injected unannealed poly-Si/thermal oxide/Si structures, respectively.
Figure 14 illustrates that the trapped charge, $\mathrm{E} \delta$ ', and $\mathrm{E}_{\gamma}^{\prime}$ centers are all spatially located throughout the oxide for the annealed structures selectively injected with holes. For the unannealed poly-Si/thermal oxide/Si structures, the trapped charge, $\mathrm{E}_{\delta^{\prime}}$ and $\mathrm{E}_{\gamma^{\prime}}$ centers are all located near the bottom $\mathrm{Si} / \mathrm{SiO}_{2}$ interface as shown in Fig. 15. (A linear relationship between the midgap voltage shift, $V_{m g}$, and the oxide thickness indicates that the net positive space charge is located near the bottom $\mathrm{Si} / \mathrm{SiO}_{2}$ interface.)

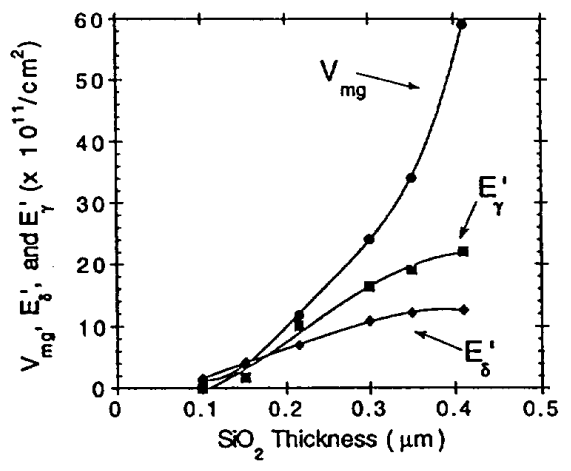

Fig. 14. Etchback experiments of the midgap voltage shift, and $\mathrm{E}_{\gamma^{\prime}}$ and $\mathrm{E}_{\delta}{ }^{\prime}$ centers in annealed poly-Si/thermal oxide/Si structures injected with holes.

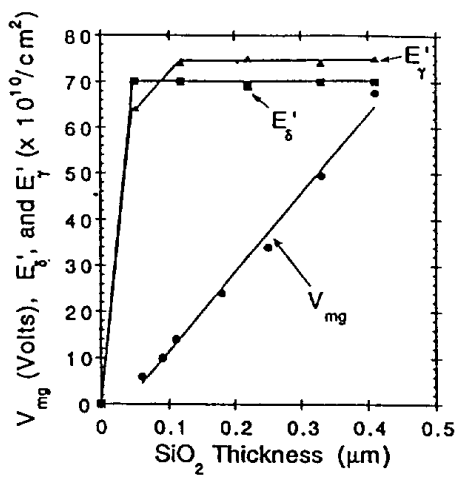

Fig. 15. Etchback experiments of the midgap voltage shift, and $\mathrm{E}_{\gamma^{\prime}}$ and $\mathrm{E}_{\delta^{\prime}}$ centers in unannealed poly-Si/thermal oxide/Si structures injected with holes.

It is admittedly perplexing why in the unannealed structure the $\mathrm{E}_{\gamma^{\prime}}$ and $\mathrm{E}_{\delta}{ }^{\prime}$ centers are spatially correlated with the positive space charge when the 
oxides are selectively injected with holes; whereas, they are not in the case of an unbiased, unfiltered VUV irradiation. The lack of a correlation between positive charge and $E^{\prime}$ centers appears to be generally observed in VUV irradiated thermal oxide films on $\mathrm{Si}$ $[31,32]$. These results further suggest that a correlation between $\mathrm{E}^{\prime}$ centers and positive charge is not always observed in thermal oxide structures, let alone SOI materials. This is certainly the general trend observed in this study.

Last, we mention that low temperature EPR experiments show that two donor defects are found in the unirradiated annealed and unannealed poly$\mathrm{Si} /$ thermal oxide/Si structures. Again, it is suggested, following our earlier arguments in the BESOI section, that these donors are O-related. The g-tensors and temperature dependence of these signals are also consistent with our assignment of these centers as $\mathrm{O}$ related. There is a substantial increase in at least one type of O-related donor the annealed $\mathrm{Si} /$ thermal oxide/Si structures. Recall that O-related donors are also observed in SIMOX [5,14,28] materials.

It becomes quite obvious at this point that the types of defects observed in the annealed $\mathrm{Si} /$ thermal oxide/Si structures and SIMOX materials are very similar. This strongly suggests that it is the high temperature post-implantation anneal during the SIMOX process that leads to much of the oxide degradation.

The physical mechanism for the degradation process of the $\mathrm{Si} / \mathrm{SiO}_{2} / \mathrm{Si}$ sandwich have been explained elsewhere by Devine et al. [19]. Simply stated, these results demonstrate that high temperature annealing creates oxygen vacancies $\left(\mathrm{E}_{\gamma^{\prime}}\right)$ and $\mathrm{Si}$ interstitial/oxygen vacancy complexes $\left(E \delta^{\prime}\right)$ in the oxide and $\mathrm{O}$-related donors in the $\mathrm{Si}$ substrates. It was suggested that, during the high temperature postoxidation anneal, oxygen vacancies and/or oxygen vacancy complexes are created in the oxide by the outdiffusion of $\mathrm{O}$. Si interstitials which are trapped at these complexes (to form $\mathrm{E}_{\delta}^{\prime}$ centers) originate at the $\mathrm{Si} / \mathrm{SiO}_{2}$ interface which is known from the oxidation studies of Tiller [33] to act as a source of $\mathrm{Si}$. The $\mathrm{O}$ released from the oxide diffuses into the bulk of the overlying $\mathrm{Si}$ substrates to generate $\mathrm{O}$-related donors or $O$ interstitials. The driving force for this reaction is the chemical potential difference of the $\mathrm{O}$ atoms in the oxide film and the Si substrate.

\section{COMPARING DEFECTS IN BESOI AND SIMOX}

Four paramagnetic defects are observed in both BESOI and SIMOX: charged oxygen vacancy $\mathrm{E}_{\gamma}$ centers, delocalized hole centers ( $\mathrm{EH}$ or $\mathbf{E} \delta^{\prime}$ centers), amorphous silicon D centers, and oxygen-related donors. It is quite amazing that the same four classes of defects are created by hole injection in both BESOI and SIMOX given their drastically different processing histories. It is suggested that some of the defects are similar because of the high temperature anneal that occurs in both processes, i.e., the O-related donors. At this point it should be stressed that most of the SOI technologies employ a high temperature anneal in a relatively inert ambient at some stage in the process. Therefore, it is suggested that all SOI technologies are likely to be susceptible to the degradation which results from the $O$ outdiffusing from the oxide layer. Similar conclusions, based on electrical measurements, were also suggested by Stahlbush et al. [1]. Another similarity worth mentioning is that delocalized hole centers are present in both materials. This is an interesting aspect of these materials since these are a relatively new class of defects. This study has elucidated an interesting aspect regarding the physical nature of these centers; they all appear to be hole traps that are less stable than the $E_{\gamma}$.

Nonetheless, there are a few noticeable differences with regard to the spatial locations of the EPR centers. For instance, the defects in BESOI materials are located near the top $\mathrm{Si} / \mathrm{SiO}_{2}$ interface, near the original bond between the oxides; whereas, the EPR centers in SIMOX are located throughout the dielectric.

\section{CONCLUSIONS}

We have combined EPR and CV measurements to identify the chemical nature and charge state of defects in BESOI, SIMOX, and poly-Si/thermal oxide/Si materials. Our results show the existence of four different paramagnetic defects common to the SIMOX and BESOI buried oxide materials, i.e., charged oxygen vacancies, delocalized hole centers, amorphous Si centers, and oxygen-related donors; the former three are all positively charged and are located in the oxide, while the latter is located in the c-Si substrate. For the SIMOX samples, the defects are all related to excess-Si. From our results on poly$\mathrm{Si} /$ thermal oxide/Si structures, it is believed that this results from out-diffusion of the implanted oxygen during the post-implantation high temperature anneal. For the BESOI materials, we note that the spatial location of the radiation-induced EPR-active defects are located at or near the bond between the seed and handle wafers. This makes this bonded interface a trap site that may lead to radiation-induced backchannel leakage, and may be an important consideration in fabricating radiation hardened BESOI wafers.

\section{AKNOWLEDGMENTS}

We would like to thank A. Stesmans and W.K. Schubert for many stimulating discussions. This work performed in part at Sandia National Laboratories was supported by the U.S. Department of Energy under contract DE-AC04-76DP00789. 


\section{REFÉRENCES}

1. R.E. Stahlbush, G.J. Campisi, J.B. McKitterick, W.P. Maszara, P. Roitman, and G.A. Brown, "Electron and Hole Trapping in Irradiated SIMOX, ZMR, and BESOI Buried Oxides," IEEE Trans. Nucl. Sci., NS-39, 2095 (1992).

2. C.A. Pennise and H.E. Boesch, Jr., "Determination of the Charge-Trapping Characteristics of Buried Oxides Using a 10-kev X-Ray Source," IEEE Trans. Nucl. Sci., NS-37, 1990 (1990).

3. A.G. Revesz, G.A. Brown, and H.L. Hughes, "Properties of Buried $\mathrm{SiO}_{2}$ Films in SIMOX Structures," MRS Symp. Proc., eds. J. Kanicki, W.L. Warren, R.A.B. Devine, and M. Matsumura, Vol. 284 (1993)

4. H.E. Boesch, Jr. and T.L. Taylor, "Time-Dependent RadiationInduced Charge Effects in Wafer-Bonded SOI Buried Oxides," IEEE Trans. Nucl. Sci., NS-39 (1992).

5. A. Stesmans, R.A.B. Devine, A.G. Revesz, and H.L. Hughes, "Irradiation-Induced EPR Active Defects in SIMOX Structures," IEEE Trans. Nucl. Sci., NS-37, 2008 (1990).

6. R.E. Stahlbush, W.E. Carlos, and S.M. Prokes, "Radiation and Processing Irduced Effects in SIMOX: a Spectroscopic Study," IEEE Trans. Nucl. Sci., NS-34, 1680 (1987).

7. R.A.B. Devine, J.-L. Leray, and J. Margail, "Ultraviolet Radiation Induced Defect Creation in Buried $\mathrm{SiO}_{2}$ Layers," Appl. Phys. Lett., 59, 2275 (1991).

8. J.F. Conley, P.M. Lenahan, and P. Roitman, "Electron Spin Resonance of Trapping Centers in SIMOX Buried Oxides," in Insulating Films on Semiconductors 1991, edited by W. Eccleston and M. Uren (Adam Higler, Bristol, 1991) p. 259, and J.F. Conley, P.M. Lenahan, and P. Roitman, "Electron Spin Resonance Study of E' Trapping Centers in SIMOX Buried Oxides," IEEE Trans. Nucl. Sci., NS-38, 1247 (1991).

9. M.E. Zvanut, R.E. Stahlbush, W.E. Carlos, H.L. Hughes, R.K. Lawrence, R. Hervey, and G.A. Brown, "SIMOX With Epitaxial Silicon: Point Defects and Positive Charge," IEEE Trans. Nucl. Sci., NS-38, 1253 (1991).

10. D. Herve, J.L. Leray, and R.A.B. Devine, "Comparative Study of Radiation-Induced Electrical and Spin Active Defects in Buried $\mathrm{SiO}_{2}$ Layers," J. Appl. Phys., 72, 3634 (1992).

11. J.F. Conley, P.M. Lenahan, and P. Roitman, "Electron Spin Resonance of SIMOX Oxides: Evidence for Structural Change and a Deep Electron Trap," Appl. Phys. Lett., 60, 2889 (1992).

12. W.L. Warren, J.R. Schwank, M.R. Shaneyfelt, D.M. Fleetwood, and P.S. Winokur, "Hydrogen Interactions with Delocalized Spin Centers in Buried $\mathrm{SiO}_{2}$ Thin Films," Appl. Phys. Lett., 62, 1661 (1993).

13. A. Stesmans and K. Vanheusden, "Depth Profiling of Oxygen Vacancy Defect Generation in Buried $\mathrm{SiO}_{2}$," MRS Symp. Proc., eds. J. Kanicki, W.L. Warren, R.A.B. Devine, and M. Matsumura, Vol. 284 (1993)

14. K. Vanheusden and A. Stesmans, "Shallow Donor in SIMOX Structures Revealed by Electric Field Modulated Electron Spin Resonance," Appl. Phys. Lett., 62, 273 (1993).

15. W.L. Warren, D.M. Fleetwood, M.R. Shaneyfelt, J.R. Schwank, P.S. Winokur, and R.A.B. Devine, "Excess-Si Related Defect Centers in Buried $\mathrm{SiO}_{2}$ Films," Appl. Phys. Lett., 62, 3330 (1993).

16. F.J. Feigl, W.B. Fowler, and K.L. Yip, "Oxygen Vacancy

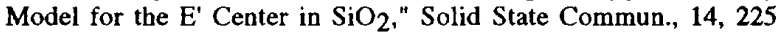
(1974).

17. D.L. Griscom, "Characterization of Three E' Center Variants in $\mathrm{X}$ - and $\gamma$-irradiated High Purity a-SiO $2, "$ Nucl. Instrum. Meth. B1, 481 (1984).

18. D.L. Griscom and E.J. Friebele, "Fundamental RadiationInduced Defect Centers in Synthetic Fused Silicas: Atomic Chlorine, Delocalized E' Centers, and a Triplet State," Phys. Rev. B34, 7524 (1986)
19. R.A.B. Devine, D. Mathiot, W.L. Warren, D.M. Fleetwood, and B. Aspar, "Point Defect Generation and Oxide Degradation During Annealing of the $\mathrm{Si}-\mathrm{SiO}_{2}$ Interface," Appl. Phys. Lett., accepted for publication.

20. W.P. Maszara, G. Goetz, A. Caviglia, and J.B. McKitterick, " Bonding of Si Wafers for Silicon-on-Insulator," J. Appl. Phys., Vol. 64, 4943 (1988).

21. The SIMOX wafers were purchased from SOITEC/USA, Peabody, MA 01960.

22. Y. Wu and A. Stesmans, "Nature of Paramagnetic Centers in aSi and a-Si:H," Phys. Rev. B38, 2779 (1988).

23. A. Stesmans, "New Intrinsic Defect in As-Grown Thermal $\mathrm{SiO}_{2}$ on (111) Si," Phys. Rev. B45, 9501 (1992).

24. K.L. Brower, "Formation and Passivation of New Paramagnetic Defects Associated with Thermal Oxides on Silicon," MRS Symp. Proc., 105, 219 (1988).

25. J.F. Conley and P.M. Lenahan, "Room Temperature Reactions Involving $\mathrm{Si}$ Dangling Bond Centers and Molecular $\mathrm{H}$ in amorphous $\mathrm{SiO}_{2}$ Thin Films on $\mathrm{Si}$," Appl. Phys. Lett., 62, 40 (1993).

26. S.H. Muller, M. Sprenger, E.G. Sieverts, and C.A.J. Ammerlaan, "EPR Spectra of Heat-Treatment Centers in Oxygen Rich Silicon," Solid State Commun., Vol. 25, 987 (1978).

27. M. Suezawa, K. Sumino, and M. Iwaizumi, "Electron Spin Resonance Study of Oxygen Donors in Silicon Crystals," J. Appl. Phys. 54, 6594 (1983).

28. A. Stesmans, A.G. Revesz, and H.L. Hughes, Electron Spin Resonance of Defects in Silicon-on-Insulator Structures Formed by Oxygen Implantation: Influence of Gamma Irradiation," J. Appl. Phys., 69, 175 (1991).

29. E.G. Sieverts, "Classification of Defects in Silicon After Their g-values," Phys. Stat. Sol. B120, 11 (1983).

30. G. Feher, "Electron Spin Resonance Experiments on Donors by the Electron Nuclear Double Resonance Technique," Phys. Rev. 114, 1219 (1959)

31. K. Yokogawa, Y. Yajima, T. Mizutani, S. Nishimatsu, and K. Suzuki, "Positive Charges and E' Centers Formed by Vacuum Ultraviolet Radiation in $\mathrm{SiO}_{2}$ Grown on $\mathrm{Si}$, "Jpn. J. Appl. Phys., 29, 2265 (1990).

32. R.A.B. Devine, "Radiation-Induced Structural Changes in amorphous $\mathrm{SiO}_{2}$ : I Point Defects," Jpn. J. Appl. Phys., 31, 4411 (1992).

33. W.A. Tiller, "On the Kinetics of the Thermal Oxidation of Silicon," J. Electrochem. Soc., 128, 689 (1981). 